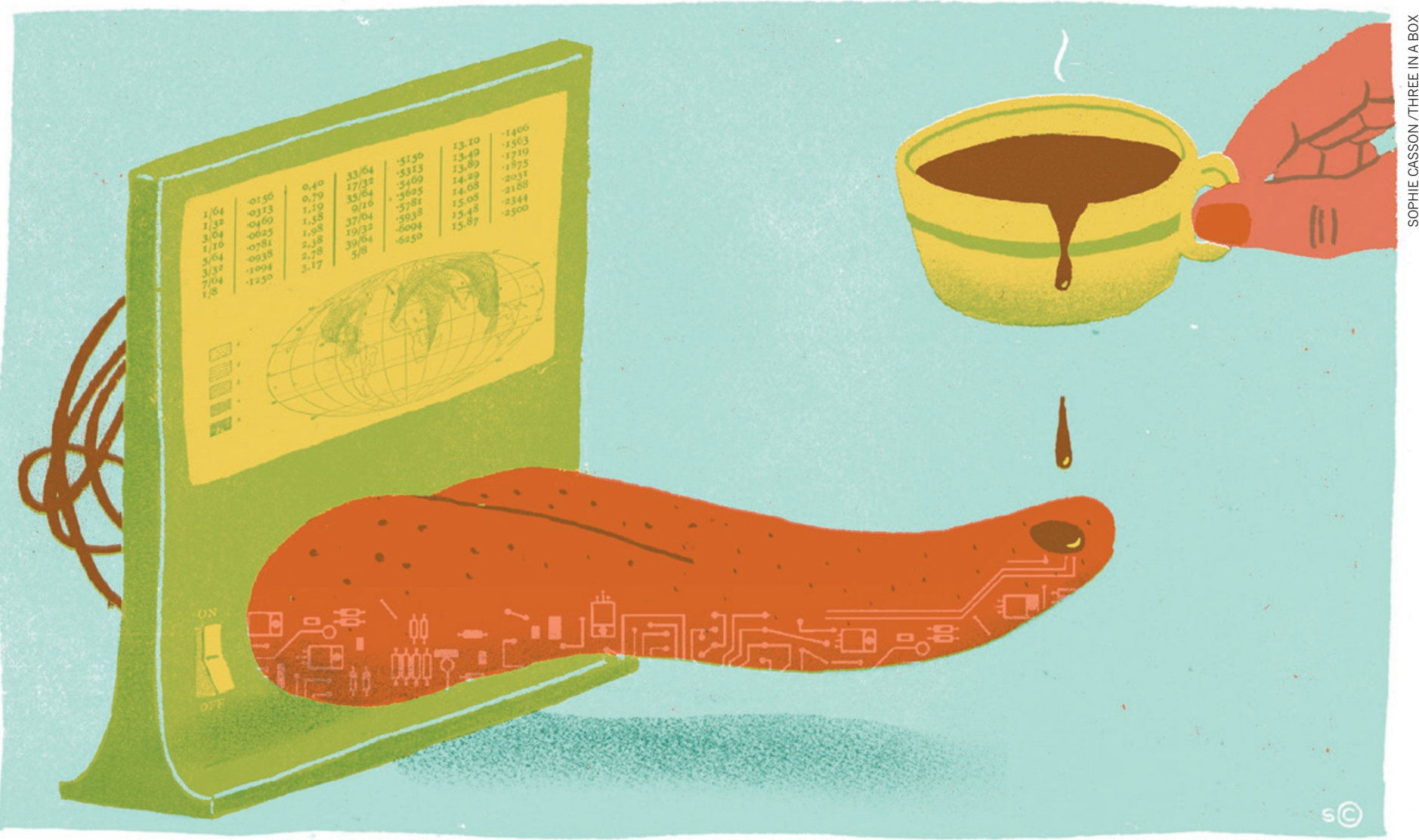

TECHNOLOGY

\title{
The taste of things to come
}

\section{Artificial tongues that mimic the human sensory experience could aid the development of better and more consistently flavoured foods.}

\section{BY NEIL SAVAGE}

I magine a food wholesaler in London deciding whether to buy a new batch of coffee from a farmer in Brazil. She'd like to taste a sample to see if it will appeal to her customers. So she links to the supplier over the Internet, makes a selection, turns to a machine on her desk, and voilà! - she's sipping coffee on another continent.

This scenario - replicating the flavour of not only coffee but a wide variety of food and drink - isn't all that far fetched, says physicist Seunghun Hong of Seoul National University in South Korea. "You can actually reproduce those smells or tastes using very cheap chemicals," he says.

What's lacking for such a device is not the technology to recreate flavours and aromas, but the data about the specifics of such sensations that could be transmitted from the Brazilian grower to the British buyer. "We don't have those kinds of quantitative numbers to evaluate your smell and taste. That's why you cannot say that this coffee tastes the same as coffee ten years ago," Hong explains.
But he and other researchers are working to change that, developing artificial tongues that can respond to flavours in ways analogous to the human tongue. Such sensors could provide quality control in food and agriculture, record information about taste in ways that are not now possible, aid the development of new flavours and artificial sweeteners, and help make medicines more palatable.

\section{ELECTRONIC TONGUES}

There are well-established technologies for measuring and reproducing three of the five human senses - sight, sound and touch — but mimicking our two chemical senses, taste and smell, has proved more challenging. Kenneth Suslick, a chemist and materials scientist at the University of Illinois at Urbana-Champaign, says that artificial versions of these senses would have practical applications and also provide an aid to understanding another aspect of human biology.

Suslick works on both artificial tongues and artificial noses: the noses deal with gases, whereas the tongues handle liquids (and solids that have been liquefied, which is what happens when we eat). Human noses, and the devices that mimic them, contain hundreds of different receptors capable of identifying the complex patterns of thousands of smells. Taste, by contrast, is made up of just a handful of components - the exact number is disputed, but researchers have agreed on at least five (see 'Hardwired for taste', page S7).

Taste lends itself to automation because it is fundamentally a chemical process. Sweetness sensors react to sugar molecules. Sourness is a measure of $\mathrm{pH}$. Saltiness is a measure of the positively charged ions of alkali metals, notably sodium. And umami, the savoury or meaty taste, is detected by a receptor for glutamate. Only the fifth major taste, bitterness, is poorly defined, and may be a catch-all term for several different chemical reactions, Suslick says.

Artificial tongues often rely on measuring changes in electrical potential or current caused by the target molecule reacting with a receptor. These devices are therefore often called electronic tongues, or e-tongues, and work in a similar way to the real thing. In the human tongue, 
․ various molecules in food bind to proteins in the taste buds, producing a pattern of electrical signals that the brain interprets as a particular flavour. Although it might be possible to build a device that would break down a food into its component chemicals and measure their relative quantities, creating such a set of instructions for say chocolate ice-cream wouldn't be practical, because a single food item might contain more than 1,000 different chemical substances. And that's not how people identify tastes anyway, says Kiyoshi Toko, professor of information science and electrical engineering at Kyushu University in Fukuoka, Japan.

"Humans don't discriminate each chemical substance," he says, but rather classify a discrete set of tastes. He believes there are two additional tastes beyond those widely accepted. One he calls astringency, which is a form of bitterness caused by tannins. The other he calls pungency - that's the sting from foods such as hot peppers, and in humans is experienced by receptors for heat and pain.

Toko has developed an e-tongue that consists of a series of polymer membranes, each coated with a different lipid, fitted onto a plastic tube and connected to an electrode. When the tube is immersed in a sample liquid, the taste molecules in the liquid interact with the lipids and change the electrical potential of the membranes in a characteristic way. The result is a readout that corresponds to taste.

A company founded to market his technology - Intelligent Sensor Technology of Kanagawa, Japan - has sold about 300 taste sensing machines in the past five years. The system can examine foods such as soy sauce, soup and sake for six taste qualities - the five standard ones plus astringency. The same sensors also produce readings for five aftertastes, mostly variations of bitterness as well as the 'richness' evoked by umami. He says he doesn't know how to make a sensor to detect pungency, but he's working on it.

Hong has a device that works in a similar way. He built a transistor using carbon nanotubes, which are highly sensitive to electrical changes, and coated the surface of the nanotubes with a protein - one that detects bitterness, for example. When substances in the sample bind to the protein, they send a signal through the transistor, and a change in electrical conductance registers the bitterness. This technique mimics the human response more closely than some other approaches, he says, because the proteins he uses are those actually found in human taste buds, which makes them highly selective for the molecules to be tasted. He produces the proteins by inserting human genes into Escherichia coli bacteria. "Our device has exactly the same response as the human taste system," he says.

Suslick's system, by contrast, relies on colourchanging dyes, rather than electrical changes. His sensors are designed to measure sweetness. He originally printed an array of commercially available reagents on an acetate membrane, measuring about $2.5 \mathrm{~cm}$ by $4 \mathrm{~cm}$. The sensor

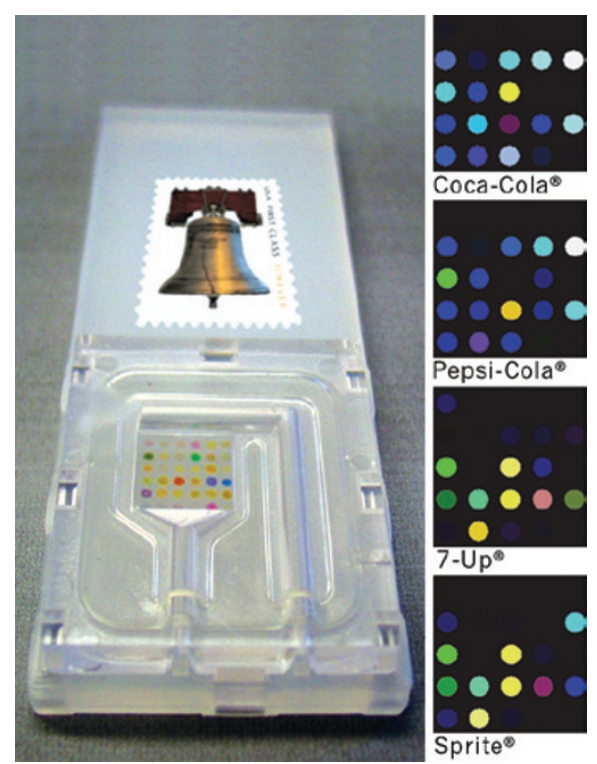

Disposable chips produce flavour 'fingerprints'.

can simply be dipped into a sample liquid, where various substances react with the different pigments and change colour. A computer compares images of the array taken before and after dipping, and produces a 'difference map' that shows how much each spot has changed.

He tested his sensor on 14 different natural and artificial sweeteners. Each array produced a pattern of colours unique to the particular sweetener, providing visible fingerprints for substances including aspartame, saccharin and fructose. The cheap, disposable sensors gave results in about two minutes, speed that could be attractive to a beverage company that wants to use the sensors for quality control. Combining this 'taste chip' with other arrays for sour, bitter, salt and umami tastes might provide a reasonable mimic of the human tongue, Suslick says.

\section{A FLAVOUR SENSATION}

However, neither e-tongues nor colour matrices can completely reproduce the experience of flavour. "An awfully large fraction of what we call taste is actually smell," Suslick points out. "Anyone who's ever had a head cold knows that." The whole taste experience is based on a complex interaction of responses by numerous receptors. Each receptor can respond to more than one chemical, and its response can affect how the brain interprets the responses from other receptors. The result, whether in the brain or an artificial system, is a complicated feedback pattern. Researchers use statistical methods from the field of artificial intelligence to map out the complex response patterns from an array of sensors. Suslick says it's not yet clear how many points such a map must include to describe an approximation of someone's reaction to taste.

"Ultimately, we would like to take the patterns and see if they can be predictive of human response," says chemist Eric Anslyn of the University of Texas at Austin. With a colleague who studies wine-making at the University of
California, Davis, Anslyn has developed a sensor that can identify different wine varieties. His device can distinguish between a Pinot Noir and a Merlot, for instance, but it cannot yet discern nuances, such as whether the drink is oaky or fruity.

A crucial part of developing an artificial tasting system is calibrating its results to real human responses. In Denmark, chemist Anders Malmendal of the University of Aarhus is trying to do this by using what he calls a 'magnetic tongue'. His technique relies on readings from nuclear magnetic resonance (NMR) spectroscopy, a common laboratory analytical technique for identifying chemical composition and concentrations. Often used in metabolomics, which screens biological samples for the by-products of metabolic activity, NMR is a good way to detect toxic effects of new drugs. Malmendal, along with colleagues from the University of Naples Federico II in Italy, used NMR spectroscopy to identify different brands of canned tomatoes. The researchers bought 18 cans at stores around Naples and used NMR to obtain their chemical signatures, each consisting of 870 variables.

To match these signatures to subjective human taste, Malmendal and colleagues turned to a panel of 12 human experts. These tasters provided detailed descriptions of flavour, odour and texture, according to an accepted set of food industry standards. Malmendal's team then matched the NMR data to the panellists' assessments, so they could use the chemical signature to say what a particular tomato would taste like to people.

With a large enough library of reference samples, food producers could test a sample and predict how consumers would react to it. One company, Bruker Biospin of Cologne, Germany, sells a juice screener that uses NMR spectroscopy and a library of more than 3,000 reference samples of 30 types of fruit from 50 countries. Quality controllers can tell if the juice is fresh or from concentrate, whether there's added sugar, and whether cheaper mandarin juice is mixed in with the orange juice - in some cases they even can tell roughly where the oranges originated. In March 2012, the company introduced a similar system for wine.

Any technique that can consistently distinguish one food sample from another can be used this way. Build up a library of human assessments of various instances of different foods, and you can predict the taste of new samples based on how well they match. But Suslick points out two problems with this approach. Paying experts to assess every variety of every food would be prohibitively expensive. And in the end, no matter how sophisticated the gadgetry, taste remains a stubbornly subjective experience. "Can we distinguish between coffees? Yes," Suslick says. "Can I tell you what the best coffee is? No."

Neil Savage is a freelance science writer based in Lowell, Massachusetts. 